International Journal of Pure and Applied Mathematics

Volume 90 No. 1 2014, 1-4

ISSN: 1311-8080 (printed version); ISSN: 1314-3395 (on-line version)

url: http://www.ijpam.eu

doi: http://dx.doi.org/10.12732/ijpam.v90i1.1

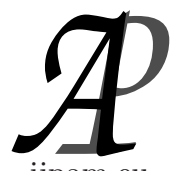

ijpam.eu

\title{
A NON-UNIFORM BOUND ON POISSON APPROXIMATION FOR INDEPENDENT BERNOULLI RANDOM SUMMANDS
}

\author{
K. Teerapabolarn \\ Department of Mathematics \\ Faculty of Science \\ Burapha University \\ Chonburi, 20131, THAILAND
}

\begin{abstract}
A non-uniform bound for the distance between the distribution of random sums of independent Bernoulli random variables and an appropriate Poisson distribution is obtained. It is sharper than the bound reported in [7].
\end{abstract}

AMS Subject Classification: 62E17, 60F05, 60G05

Key Words: Bernoulli random variable, non-uniform bound, Poisson approximation, random sums

\section{Introduction}

Let $X_{1}, X_{2}, \ldots$ be a sequence of independent Bernoulli random variables, each with probability $p_{i}=P\left(X_{i}=1\right)=1-P\left(X_{i}=0\right)$, and let $N$ be a non-negative integer-valued random variable and independent of the $X_{i}$ 's. Let us consider the random sums $S_{N}=\sum_{i=1}^{N} X_{i}$ and $N=n \in \mathbb{N}$ is fixed, there has been some research on topics related to the approximation of the distribution of $S_{n}$ by an appropriate Poisson distribution, which can be found in [1], [2], [3]-[6], etc. Let $\lambda_{N}=\sum_{i=1}^{N} p_{i}, \lambda=E\left(\lambda_{N}\right)$ and $U_{\lambda}$ a Poisson random variable with mean $\lambda$. For approximating the distribution of $S_{N}$, Yannaros [7] gave a uniform bound for the distance between the distribution of $S_{N}$ and $U_{\lambda}$ as follows:

Received: July 17, 2013

(c) 2014 Academic Publications, Ltd. url: www.acadpubl.eu 


$$
\left|P\left(S_{N} \in A\right)-P\left(U_{\lambda} \in A\right)\right| \leq E\left|\lambda_{N}-\lambda\right|+E\left(\frac{1-e^{-\lambda_{N}}}{\lambda_{N}} \sum_{i=1}^{N} p_{i}^{2}\right)
$$

where $A \subseteq \mathbb{N} \cup\{0\}$. It is observed that this bound is uniformly in $A$, which does not depend on $A$.

In this study, we are interest to determine a non-uniform bound of (1.1), which is in Section 2. Concluding remarks are presented in the last section.

\section{Results}

The following theorem presents a non-uniform bound for the distance between the distributions of $S_{N}$ and $U_{\lambda}$, which is the desired result.

Theorem 2.1. For $A \subseteq \mathbb{N} \cup\{0\}$, then we have

$$
\begin{aligned}
\left|P\left(S_{N} \in A\right)-P\left(U_{\lambda} \in A\right)\right| \leq \min \left\{1, \sqrt{\frac{2}{e \lambda}}\right\} E\left|\lambda_{N}-\lambda\right| \\
+\min \left\{E\left(\frac{1-e^{-\lambda_{N}}}{\lambda_{N}} \sum_{i=1}^{N} p_{i}^{2}\right), \frac{1}{x_{A}} E\left(\sum_{i=1}^{N} p_{i}^{2}\right)\right\},
\end{aligned}
$$

where $\frac{1}{x_{A}}$ is taken to be 1 when $x_{A}=0\left(x_{A}^{\star}=0\right.$ or $\left.x_{A}^{*}=1\right)$ and for $x_{A}>0$, it is given by

$$
\frac{1}{x_{A}}= \begin{cases}\frac{1}{x_{A}^{\star}} & \text { if } 0 \in A, \\ \frac{1}{x_{A}^{*}-1} & \text { if } 0 \notin A,\end{cases}
$$

and $x_{A}^{*}=\min \{x \mid x \in A\}$ and $x_{A}^{\star}=\max \{x \mid\{0, \ldots, x\} \subseteq A\}$.

Proof. It follows the fact that

$$
\begin{aligned}
\left|P\left(S_{N} \in A\right)-P\left(U_{\lambda} \in A\right)\right| \leq & \sum_{n=0}^{\infty} P(N=n)\left|P\left(S_{n} \in A\right)-P\left(U_{\lambda} \in A\right)\right| \\
\leq & \sum_{n=0}^{\infty} P(N=n)\left|P\left(S_{n} \in A\right)-P\left(U_{\lambda_{n}} \in A\right)\right| \\
& +\left|P\left(U_{\lambda_{N}} \in A\right)-P\left(U_{\lambda} \in A\right)\right|
\end{aligned}
$$


Applying the corresponding results in [4] and [1], we obtain

$$
\left|P\left(S_{n} \in A\right)-P\left(U_{\lambda_{n}} \in A\right)\right| \leq \min \left(\frac{1-e^{-\lambda_{n}}}{\lambda_{n}} \sum_{i=1}^{n} p_{i}^{2}, \frac{1}{x_{A}} \sum_{i=1}^{n} p_{i}^{2}\right)
$$

and

$$
\left|P\left(U_{\lambda_{N}} \in A\right)-P\left(U_{\lambda} \in A\right)\right| \leq \min \left\{1, \sqrt{\frac{2}{e \lambda}}\right\} E\left|\lambda_{N}-\lambda\right|,
$$

respectively. Taking these bounds into (2.2), hence (2.1) is obtained.

If $X_{i}$ 's are identically distributed, then the following corollary is an immediately consequence of the Theorem 2.1

Corollary 2.1. Let $A \in \mathbb{N} \cup\{0\}$, if $p_{1}=p_{2}=\cdots=p$, then we have the following:

$$
\begin{aligned}
&\left|P\left(S_{N} \in A\right)-P\left(U_{\lambda} \in A\right)\right| \leq \min \left\{E\left(1-e^{-p N}\right) p, \frac{p^{2} E(N)}{x_{A}}\right\} \\
&+\min \left\{1, \sqrt{\frac{2}{e E(N) p}}\right\} p E|N-E(N)| .
\end{aligned}
$$

Remark. We consider the following facts:

1. $\min \left(\frac{1-e^{-\lambda_{N}}}{\lambda_{N}}, \frac{1}{x_{A}}\right) \leq \frac{1-e^{-\lambda_{N}}}{\lambda_{N}}$ and

2. $\min \left\{1, \sqrt{\frac{2}{e \lambda}}\right\} E\left|\lambda_{N}-\lambda\right| \leq E\left|\lambda_{N}-\lambda\right|$.

Thus, the bound in (2.1) is sharper than the bound in (1.1).

\section{Conclusion}

In this study, a non-uniform bound for the distance between the distribution of random sums of independent Bernoulli random variables and an appropriate Poisson distribution was determined. By comparing, this bound is sharper than that reported in [7]. Thus, it is more appropriate for measuring the accuracy of the approximation. 
K. Teerapabolarn

\section{References}

[1] A.D. Barbour, L. Holst, S. Janson, Poisson Approximation, Oxford Studies in Probability 2, Clarendon Press, Oxford, 1992.

[2] K. Neammanee, Pointwise Approximation of Poisson binomial by Poisson distribution, Stochastic Modelling and Applications, 6 (2003), 20-26.

[3] C.M. Stein, Approximate Computation of Expectations, IMS, Hayward California, 1986.

[4] K. Teerapabolarn, New non-uniform bounds on Poisson approximation for dependent Bernoulli trials, To appear in: Bulletin of the Malaysian Mathematical Sciences Society.

[5] K. Teerapabolarn, K. Neammanee, Poisson approximation for sums of dependent Bernoulli random variables, Acta Mathematica Academiae Paedagogicae Nyiregyhaziensis, 22 (2006), 87-99.

[6] K. Teerapabolarn, T. Santiwipanont, Two non-uniform bounds in the Poisson approximation of sums of dependent indicators, Thai Journal of Mathematics, 5 (2007), 15-39.

[7] N. Yannaros, Poisson approximation for random sums of Bernoulli random variables, Statist. Probab. Lett., 11 (1991), 161-165. 